\title{
Miranda
}

Revue pluridisciplinaire du monde anglophone /

Multidisciplinary peer-reviewed journal on the English-

speaking world

$12 \mid 2016$

Mapping gender. Old images ; new figures

\section{Le Common Law est une femme... et quelle femme !}

Isabelle Richard

\section{OpenEdition}

\section{Journals}

Édition électronique

URL : https://journals.openedition.org/miranda/8686

DOI : $10.4000 /$ miranda.8686

ISSN : 2108-6559

Éditeur

Université Toulouse - Jean Jaurès

Référence électronique

Isabelle Richard, « Le Common Law est une femme... et quelle femme! », Miranda [En ligne], 12 | 2016, mis en ligne le 02 mars 2016, consulté le 21 septembre 2021. URL : http://journals.openedition.org/ miranda/8686 ; DOI : https://doi.org/10.4000/miranda.8686

Ce document a été généré automatiquement le 21 septembre 2021.

\section{$\Theta \Theta \Theta \Theta$}

Miranda is licensed under a Creative Commons Attribution-NonCommercial-NoDerivatives 4.0 International License. 


\title{
Le Common Law est une femme... et quelle femme!
}

\author{
Isabelle Richard
}

\section{Introduction}

1 En anglais comme en français, le concept de Common Law est généralement associé au féminin, soit sur le plan grammatical (pour le français), soit sur celui de la représentation mentale (pour l'anglais). En français, il est traditionnellement admis par la communauté scientifique, et par celle des traducteurs juridiques, que le terme Common Law ne se traduit pas, car il ne fait référence à aucun concept connu dans notre propre système de droit, qui appartient en effet à une famille différente, celle des droits romano-germaniques. Il va sans dire que le calque constituerait un contresens majeur, notre «droit coutumier» ou notre «droit commun » étant totalement distincts du concept de Common Law. Ce dernier peut se définir comme «l'ensemble des règles de droit en vigueur dans les pays de culture juridique anglo-saxonne », explique Picotte dans son Juridictionnaire $(2014,1040)$. Une fois ceci établi, se pose la question de l'article à utiliser devant cet emprunt. Généralement, il est de genre féminin («la » Common Law), aussi bien en France qu'au Québec : «Au Canada, le Comité de normalisation de la terminologie française de la common law a tranché en faveur de la forme féminine en raison de sa prédominance dans l'usage ", précise Picotte $(2014,1038)$. Les raisons de ce choix en français sont essentiellement liées à la proximité phonique entre «loi » et «law».

2 En anglais, la féminisation a également lieu mais elle est liée à un autre facteur : celui de la perception que l'on a de ce concept. Il semblerait, en effet, qu'il soit associé à une figure féminine depuis que Sir Frederick Pollock, historien reconnu du droit anglais (1845-1937), a donné ses conférences intitulées The Genius of the Common Law, à l'Université de Columbia (New York) en 1911. L'objectif de cet auteur était de retracer l'histoire de cette partie essentielle du droit anglais. Dès la première page de son intervention, la personnification créée dans le titre («genius») est sexualisée par le 
biais de la métaphore "Our Lady the Common Law ", utilisée ensuite à maintes reprises tout au long du texte. Publiées l'année suivante par les Presses de cette Université, ces conférences ont connu un tel succès, notamment grâce à la renommée de leur auteur ${ }^{1}$, qu'elles ont, semble-t-il, profondément marqué l'imaginaire des juristes des deux côtés de l'Atlantique, comme en attestent un certain nombre de publications et de discours écrits depuis. Ainsi, en 1928, le juge Cardozo² a prononcé un discours intitulé «Our Lady of the Common Law » à la Saint John's Law School ; plus récemment, un ouvrage portant le même titre a été publié en 1987 par l'auteur américain Richard A. Cosgrove ; on peut également citer la conférence de Lord Justice Laws, en mars 2012, intitulée elle aussi « Our Lady of the Common Law ». En outre, dans un grand nombre d'autres écrits au titre moins évocateur, ce concept est associé à des références féminines. "La " Common Law semble bien avoir acquis un genre dans le monde anglophone.

Étymologiquement toutefois, cette féminisation n'est soutenue par aucune preuve linguistique. La féminisation, dans les conférences de Pollock est une question de représentation mentale, le but visé étant sans doute de véhiculer certains aspects de ce droit jurisprudentiel afin de mettre en valeur ce qui paraissait essentiel à leur auteur. C'est, d'ailleurs, le propre de toute métaphore : donner accès à un concept abstrait de manière partielle, et parfois partiale, grâce à une analogie avec un élément concret et/ ou familier.

4 Je me propose d'analyser la manière dont le concept de Common Law est décrit dans les conférences de Pollock, et de montrer comment cet auteur crée une représentation féminine particulière du concept de Common Law. Je compléterai cette analyse par celle de l'ouvrage de Gary Watt, intitulé Equity Stirring - The Story of Justice Beyond Law, publié en 2012. La raison de ce rapprochement est que, d'une part, les notions de Common Law et d'Equity constituent les deux sources du droit jurisprudentiel au Royaume-Uni (case law) et, d'autre part, qu'elles sont présentées, dans ces deux ouvrages, au moyen de métaphores féminines. En effet, Equity est associée à des images féminines (entre autres représentations), notamment en tant que sœur, et ancienne rivale, de «la » Common Law (l'article féminin est ici employé à dessein). Il en va de même de la Justice (Lady Justice), dont le bras armé (par l'épée) et protecteur (grâce au bouclier) garantit l'application des règles de sa mère (la Common Law). Cette féminisation d'aspects fondamentaux du droit anglais construit une rhétorique au sein de laquelle une certaine image du féminin est devenue la norme; une norme porteuse de symboles de puissance généralement associés au principe masculin. Les ayant absorbés et faits siens, elle érige un nouveau genre qui concentre en une seule entité les catégories féminin/ masculin, et le place au centre de la rhétorique juridique.

Je vais, pour commencer, expliciter les raisons pour lesquelles j'utilise l'article masculin devant le syntagme Common Law. Ensuite, je proposerai une analyse de la manière dont Pollock présente ce droit dans ses conférences, puis je discuterai de l'impact que l'image ainsi véhiculée a pu avoir sur la perception de ce concept, avant de montrer pourquoi l'emploi du féminin devrait être réservé aux contextes dans lesquels la métaphore féminine joue un rôle essentiel.

\section{Pourquoi « le » Common Law}

6 Remontons quelques siècles en arrière, au moment de l'émergence progressive du système de Common Law au Royaume-Uni. Lorsque Guillaume II, duc de Normandie, a 
conquis la Couronne d'Angleterre au $11^{\text {ème }}$ siècle, il a instauré des "cours royales " (royal courts) pour imposer la justice du roi, sans pour autant démanteler les «tribunaux locaux " (local courts) qui existaient à l'époque. Cette création a généré un système jurisprudentiel double dans lequel les deux niveaux de juridiction se distinguaient par deux caractéristiques: la première est qu'on n'y jugeait pas les mêmes types d'affaire ; la seconde est qu'on n'y parlait pas la même langue. En effet, on s'exprimait en anglais dans les « tribunaux locaux » tandis que deux langues étrangères étaient utilisées dans les " cours royales » : le latin et le franco-normand. Le latin était, entre autres, la langue dans laquelle étaient rédigés les writs, sortes de lettres indispensables à toute action en justice en Common Law. Ces documents étaient rédigés par des «clercs » (dont dérive le substantif clerk), c'est-à-dire des ecclésiastiques, qui s'exprimaient en latin et étaient les seuls, à l'époque, à maitriser l'écriture. Ainsi, durant la période médiévale, la jurisprudence des «cours royales» a été consignée dans des recueils appelés Year Books (1260-1535), en français (qui était la langue des débats) et/ou en latin. Au fil du temps, cette jurisprudence s'est imposée dans tout le pays, constituant un droit « commun » à tout le territoire (par opposition à celui né des «tribunaux locaux », considéré comme un «droit spécial»), d'où le nom de Common Law (qu'on ne pourra donc confondre ni avec le «droit commun», ni avec le «droit coutumier » français).

7 Le concept de Common Law fait bien référence à un «droit " (de genre grammatical masculin en français) et non pas à un ensemble de « lois » (ces dernières ne constituant qu'une source de droit parmi d'autres dans le système de Common Law). C'est une des raisons pour lesquelles le comparatiste Pierre Legrand défend, dans son article « Pour le Common Law " (1992), l'emploi de l'article masculin pour déterminer le syntagme Common Law. On notera qu'en espagnol et en italien, on emploie également le masculin, bien qu'en espagnol le mot «ley» soit féminin, tout comme «legge» en italien, ce qui montre que la proximité phonique entre «loi » et «law» (dont résulterait le fait qu'on utilise le féminin en français) n'est pas un argument bien convaincant, puisque cette proximité se retrouve avec «ley» aussi bien qu'avec «legge». Sur le plan conceptuel, donc, il n'y a aucune hésitation à avoir : le Common Law est un droit, et constitue, d'ailleurs, une famille à lui tout seul (on parle de la famille des droits de Common Law, également nommés droits anglo-saxons, comme on parle de celle des droits romanogermaniques, par exemple).

D'autres justifications en faveur du masculin, grammaticales cette fois, peuvent être avancées. L'une d'elles est que les expressions qui ont donné naissance au syntagme Common Law étaient soit au masculin, soit au féminin, soit au neutre, comme le montre le tableau ci-dessous.

\begin{tabular}{|c|c|c|}
\hline Féminin ${ }^{\not}$ & Neutre & Masculin \\
\hline $\begin{array}{l}\text { Lex communis }{ }^{\pi} \\
\text { Commune lei }{ }^{\pi} \\
\text { Lay commune } \\
\text { Commune ley } \\
\text { Commen ley }\end{array}$ & Ius commune & $\begin{array}{l}\text { Commun dreit } \\
\text { Commune droit } \\
\text { Comen ley }\end{array}$ \\
\hline
\end{tabular}

9 Les premiers emplois du terme Common Law datent du $14{ }^{\text {ème }}$ siècle, selon Pollock \& Maitland $(1968,176)$ mais ils étaient nettement moins fréquents que ceux de lex communis et de commune lei, ajoutent ces auteurs. Le tableau montre que les diverses formulations utilisées, qui sont issues des deux langues dans lesquelles le Common Law 
était exprimé du $12^{\text {ème }}$ au $18^{\text {ème }}$ siècles, c'est-à-dire le latin et le franco-normand (puis l'anglo-normand) $)^{3}$, étaient majoritairement au féminin. Il semblerait qu'elles étaient aussi le plus fréquemment utilisées. Parmi elles, la formulation commune ley, venant du franco-normand, aurait été prédominante durant la période allant du $13^{\text {ème }}$ aux $16^{\text {ème }}$ siècles, ce que confirme la consultation des Year Books précise Legrand $(1992,943)$.

Les Anglais, à l'époque où la Law French était la langue du droit, disaient la

Common Law, ou la commune ley. (Kerby 1982, 7)

Néanmoins, cette remarque sur la fréquence d'emploi des formulations féminines n'est pas un argument en faveur de l'emploi du féminin aujourd'hui, comme le soutient d'ailleurs très justement Legrand, étant donné que l'emploi du masculin et du féminin était fluctuant à l'époque ${ }^{4}$. En outre, même si le féminin l'a emporté fut un temps, cela ne fige pas l'emploi de l'article car « un grand nombre de substantifs ont changé de genre au cours de l'histoire ", rappelle Legrand $(1992,945)$ citant Le Grand Robert de la langue française (article « genre »). Par conséquent, l'usage, en la matière, n'est pas un facteur fiable.

11 Je retiendrai, en revanche, le propos de Bullier (2007) qui, dans son ouvrage pourtant intitulé La Common Law, discute de l'emploi du masculin et du féminin, et explique en page 2 que le masculin peut se justifier par le fait que «law» est neutre en anglais; or, en français le neutre appelle le masculin, qui est le genre grammatical non marqué.

12 Ainsi, pour des raisons prioritairement conceptuelles et, secondairement, grammaticales, il me semble que le masculin devrait être la règle en français devant ce syntagme. Sur le plan symbolique en revanche, il en va tout autrement si l'on se base sur les conférences de Pollock ${ }^{5}$.

\section{La Common Law : reine, déesse et guerrière}

13 Le nom "genius ", dans le titre, génère d'emblée la personnification de Common Law, en l'accompagnant de l'idée de succès. Très rapidement, il va s'avérer que cette personnification est féminine et que les succès sont d'ordre guerrier. Il suffit, pour cela, de lire les huit titres de la table des matières :

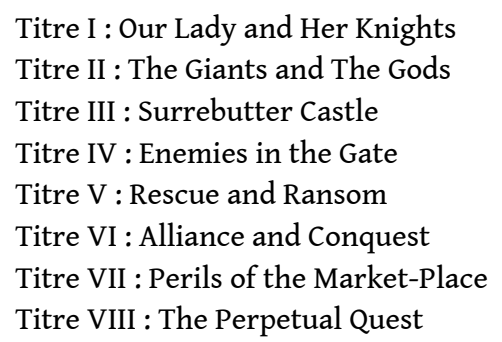

Le titre I la présente comme une reine. Le titre II introduit l'idée de divinité, même si elle n'est pas encore directement associée à la Common Law. Les titres III à VIII évoquent tous la conquête guerrière ${ }^{6}$. La Common Law n'est donc pas n'importe quelle femme. C'est une femme en armes et conquérante à laquelle, culturellement, on est tenté d'associer des qualités dites «masculines ». Cette image est présente tout au long du texte et impose, de ce fait, une représentation féminine qui en exclut toute autre et s'arroge ainsi tout l'espace symbolique, en formant une sorte de « centre » immense qui dévore et anéantit tout ce qui n'est pas lui. Voici, en illustration, une citation très représentative de la puissance qu'elle déploie : 
the Common Law shows an assimilative power which, to all appearance, grows by

what it feeds on. (44) des enfants et joue un rôle maternel protecteur (j'y reviendrai). Les hommes sont des valets dévoués qui la vénèrent, à l'exception des rois (les juges, également, remplissent un rôle particulier). Les seules autres figures féminines présentes dans ce texte sont Equity et Canon Law, contre lesquelles elle a lutté et auxquelles elle a fini par s'imposer. Cette femme guerrière est également pourvue d'autres qualités qui la placent, aux yeux des hommes (au sens large, cette fois), en position dominante.

\section{Une reine doublée d'une déesse} trois pages ; «Our Lady», quinze fois. Cela fait un total de quarante-quatre occurrences, soit une toutes les pages et demie. Pour comparaison, « genius » apparaît deux fois dans le corps du texte, aux pages 41 et 48 . Ce décalage montre que l'intérêt est clairement porté sur «lady». La Common Law possède d'ailleurs les attributs de toute reine : elle a un trône (enthroned se trouve aux pages 7 et 33 ) ; un royaume (reign à la page 23 , realm aux pages 33 et 40 ); des champions (knights aux pages 4 et 40 , champions à la page 26 , fighting the battle à la page 44); des serviteurs (officers à la page 51, servants aux pages 34 , 35,52 et 65 , to serve à la page 6 , suitors à la page 35 ); des gens qu'elle prend à son service sont mentionnés à la page 47 .

De surcroît, on la vénère comme une déesse : worship apparaît à la page 6 , our worship to her à la page 67, et my honour and worship à la page 35 ; on lui rend hommage (6); on a foi en elle (page 6); on la qualifie de «déesse » (6) que l'on compare aux dieux d'Homère (6) qui combat sans jamais faillir l'Hydre de Lerne (61). Cette dernière remarque met en lumière un autre élément caractérisant de la Common Law : elle est une guerrière.

\section{La guerrière}

Le vocabulaire de la guerre, des conflits, des armes, des batailles, des dangers et des ennemis est de loin le plus largement utilisé dans ce texte. Il est omniprésent du début à la fin de l'ouvrage et représente le champ sémantique le plus développé aussi bien en fréquence qu'en variété des mots employés. Au total, on compte cinquante-cinq occurrences de lexies relatives à ce champ sémantique, soit presque une par page :

armed / weapon / arms of precision / arms / army

battle / conflict / physical combat / strife

fight / fighting

enslaved

attack / imperil peace / hostile powers

enemy / foes / rivals

dangers / hostility

champions / knights

arduous adventure

wage a new war / warfare

triumphs / successes / conquest / success and failure

allies / companions 
La Common Law symbolise ainsi l'alliance de la puissance et du sacré. Elle est présentée comme un être qui, depuis sa naissance, s'est battu pour survivre, puis exister, et enfin s'imposer. Pollock évoque sa naissance et sa petite enfance dès la page 16, en précisant que ses pères nourriciers ("nursing fathers »), c'est-à-dire les rois, y ont joué un rôle prépondérant. En effet, le système de Common Law correspond, initialement, à la justice du roi (voir supra); une justice qui a remplacé peu à peu les anciennes coutumes du droit anglo-saxon appliqué dans les tribunaux locaux, ce que Pollock ne manque pas de rappeler :

Whatever we may think of the king's new justice as it stood between six and seven centuries ago, comparing it with all that we have learnt and accomplished since, there is no doubt that it was immensely more rational than the prehistoric methods it supplanted. (17)

Lorsqu'il décrit la Common Law comme une déesse guerrière, Pollock avait peut-être en tête une autre déesse, à savoir Pallas/Athéna (ou bien Minerve). Voici ce qu'écrit l'auteur :

She is no placid Madonna sitting in a rose garden; rather, she is like the Fortitude of the Florentine master, armed and expectant, her battle-mace lightly poised in fingers ready to close, at one swift motion, to the fighting grasp. (6)

On pourrait aisément imaginer la Common Law sous les traits de Pallas/Athéna, déesse de la guerre, des stratégies militaires et de la sagesse dans le monde antique, et l'identifier aux diverses représentations faites de cette déesse ${ }^{7}$.

Un autre point commun mérite d'être souligné : Athéna se revendique sans mère. Metis a en effet été avalée par Zeus avant qu'elle ne la mette au monde. "Je n'ai pas de mère qui m'ait enfantée » fait dire Eschyle à Athéna dans les Euménides ${ }^{8}$. De son côté, Homère dans l'Iliade (chant V) écrit : « Zeus, père [...] contre toi, tous nous combattons : car tu as engendré une fille insensée, funeste, qui toujours a l'idée d'actes abominables » (page 97). Bien entendu, nul doute que Pollock ne garderait pas la fin de cette citation, mais on retiendra l'absence de mère d'une part, et la mise en valeur du (ou des) père(s) d'autre part, comme point commun entre la déesse Athéna et la Common Law. On peut en effet penser aux "nursing fathers " de la Common Law (dont Pollock, d'ailleurs, n'évoque jamais la mère), autrement dit les rois, le plus important desquels est sans doute Henri II, souvent présenté comme celui qui a établi les principes fondateurs de ce droit par le biais de réformes qui en ont profondément marqué l'esprit (Pollock \& Maitland 1968, 82). La Common Law serait sortie de sa tête comme Athéna du crâne de Zeus, armée de sa lance et de son bouclier, et poussant un cri de guerre. En outre, le lien entre la Common Law et Athéna semble trouver un écho dans les comparaisons que fait Pollock entre la Common Law et une déesse d'une part, et la Common Law et une Madonne (Athéna étant une déesse vierge) d'autre part.

Cette remarque conduit à une autre caractéristique de la Common Law telle que la décrit Pollock.

\section{La Common Law : femme asexuée et mère protectrice}

Pollock évoque rapidement l'enfance de la Common Law (16) avant de faire un bond dans le temps, seize pages plus loin (32), quand il mentionne son âge respectable ( $a$ shrewd old lady»). Il évoque ensuite quelques caractéristiques qui indiquent qu'elle est une femme (presque) comme les autres : elle parle $(35,39)$; elle sourit $(36,39)$; d'autres 
verbes sont également utilisés qui appellent un sujet grammatical humain, comme know et make objection to (page 44), ainsi que des noms comme acquisition (46); elle a des bras, bien que Pollock ne parle que d'un seul bras capable d'atteindre des lieux au-delà des mers (page 40), ce qui souligne une fois de plus la puissance qui la caractérise, et fait penser à cette autre métaphore très fréquente dans le lexique juridique : « the long arm of the law» (qui permet d'évoquer le fait que nul ne peut échapper à la loi); elle a également des mains (59). Dans la mesure où bras et mains sont des symboles du pouvoir de l'humain sur son environnement, on peut supposer que Pollock a mentionné ces attributs physiques à dessein.

\section{Femme asexuée}

Ils prennent d'autant plus de relief que, sur le plan physique, il n'est question de rien d'autre. Aucune autre partie du corps n'est citée, certainement aucun détail qui pourrait la sexualiser. La fonction maternelle de la Common Law n'est pas organique mais protectrice. Pollock ne mentionne que ce qui semble être, pour lui, l'essentiel, à savoir: "l'affection constante de la Common Law " ("the constant affection of the Common Law", 10) pour les enfants qu'elle protège et adopte. "Her cloak will open as wide as the Madonna's, and the children she welcomes under it are adopted for her very own", explique-t-il page 44. Dans cet énoncé, la Common Law est explicitement comparée à la Madonne, c'est-à-dire encore une figure féminine, non sexualisée et sacrée.

En outre, simplement en tant que femme, elle inspire le respect. Pour certains auteurs, ce serait la raison essentielle de la féminisation forgée par Pollock qui, très tôt dans son texte, pose la question suivante (10): «Who taught us to respect women? ?. Énoncée de la sorte, cette question valide la relation [we/respect women], la présentant comme non remise en cause, de sorte que ce constat ne peut faire l'objet d'aucun doute.

Lorsque l'on combine cette image de la femme avec celle de la guerrière, le respect se teinte de crainte. Or, ce respect serait mis à mal, voire anéanti, si la description de Pollock s'attardait sur des détails anatomiques. C'est pourquoi, on peut le supposer, il se garde bien de décrire plus avant la Common Law sur le plan physique. D'autres auteurs, toutefois, ont sauté le pas et filé la métaphore. Par exemple, ce juge anglais qui a écrit qu'elle n'est pas ménopausée et tout à fait à même d'enfanter ("The common law has not suffered a menopause and is not past the age of child bearing ", cité par Galonnier 2000, \$27), toute « vieille dame » qu'elle soit. C'est une manière de souligner son extraordinaire créativité et la vitalité étonnante dont elle fait preuve, ce qui confirme, par la même occasion, son statut hors du commun.

\section{Mère féconde et protectrice}

27 La fécondité de ce système de droit tient à la manière dont il fonctionne : ses enfants sont en effet les jugements prononcés par les juges. On dit d'ailleurs de ce droit qu'il est créé par les juges ( a judge-made law ») grâce aux décisions qu'ils prennent. On peut en conclure que ses enfants font partie d'elle. D'une certaine façon, elle est ses enfants et ses enfants sont elle. Plus elle en a, c'est-à-dire plus le nombre de jugements rendus est important, plus elle grandit et se renforce, ceux qui constituent des précédents étant à leur tour des créateurs indirects. La Common Law symbolise ainsi une forme de 
parthénogenèse puisque le système qu'elle représente est intrinsèquement conçu pour se féconder lui même.

On note que Watt évoque lui aussi un élément maternel indissociable d'Equity, l'autre source de droit jurisprudentiel, dont les enfants sont «les jugements en Equity» ("Equity rulings»). Cet auteur indique que la nature profonde d'Equity est d'éduquer et, notamment, de veiller à ce que " The law " (c'est-à-dire " the Common law " dans ce contexte) reste dans le droit chemin. Cette remarque a trait à l'évolution historique d'Equity et de Common Law. La première, après avoir été la rivale de sa sœur, est finalement devenue son alliée ("Their days of strife are over", indique Pollock à la page 44). Cette rivalité s'explique par le fait que le système d'Equity a été créé pour pallier les manques et les rigidités du Common Law qui s'était figé dans des règles de jurisprudence très strictes en raison, notamment, du système de writs mentionné plus haut : aucune action n'était possible sans ce document. Il fallait donc identifier le bon writ, en fonction du préjudice qu'on estimait avoir subi et de la réparation que l'on souhait obtenir ; s'il n'en existait pas, aucune solution ne s'offrait. Equity, en revanche, était censée être flexible (31) :

Equity is always compromising in a way which tends to make the law better because it closes the gap between the rigid law and the variable contours of life.

Les qualités des deux sœurs se complètent dans le souci d'offrir la meilleure justice possible. D'ailleurs, on lit tantôt que la Justice (Lady Justice : encore une femme) est la fille d'Equity, tantôt qu'elle est celle de la Common Law. La complémentarité et l'alliance des deux sœurs a été instaurée par deux lois, votées respectivement en 1733 et 1735 (The Judicature Acts), qui permettent de demander un recours en Equity comme en Common Law dans un même tribunal, au cours de la même instance. Jusque-là, les recours devaient être demandés séparément.

Les métaphores anthropomorphiques mentionnées par Watt sont tout à fait révélatrices du type de lien qui existe entre ces deux alliées. Equity est décrite comme : "the soul and spirit of the law» (48) ainsi que sa "conscience» $(48,52,55)$. Le nom " conscience » mérite d'être souligné car il rappelle l'origine de la création d'Equity: elle s'est développée sous l'impulsion du Lord Chancellor, considéré comme «the Keeper of the King's Conscience ». C'est sous sa présidence que se tenaient les Courts of Chancery (ou Courts of Equity) qui appliquaient les principes dits « de justice » et d'" équité ». Watt précise en outre que, parmi les métaphores les plus utilisées pour parler d'Equity, se trouve celle de la personnification. Il cite ainsi un certain nombre de maximes dans lesquelles sont employés des verbes comme see, look, assist, do, abhor, allow, regard etc. ( 94) qui appellent un sujet grammatical animé.

31 Comme la Common Law, Equity est une femme de pouvoir. Pourtant, elle est à son service : «Equity is (...) servant to the law » (102), « Equity follows the law » $(94,104)$. Mais elle la suit comme un cavalier suit son cheval : « the general law is the horse and equity is its rider» (103). La cavalière (Equity) n'intervient que si sa monture (Common Law) s'écarte du droit chemin, mais c'est bien la cavalière qui dirige. D'ailleurs, lorsque conflit il y a entre Equity et Common Law, c'est la première qui prévaut (« in case of conflict, Equity shall prevail $»^{9}$ ).

32 Pollock, bien évidemment, ne présente jamais ainsi le lien qui unit Common Law et Equity, puisqu'il y aurait conflit entre les deux métaphores (déesse guerrière selon l'une, monture selon l'autre), mais aussi, et surtout, parce que l'on perdrait tous les bénéfices de la sienne. Il préfère évoquer l'existence de deux systèmes parallèles 
lorsque Equity a émergé, ce qui a généré des conflits : «On certain points there was definite conflict ", écrit-il (44). Lorsqu'il y a eu rapprochement entre ces deux sources du droit, Pollock utilise une métaphore domestique : «Equity (who) has at last come to keep house with her in England » (44).

Il demeure donc résolument dans la personnification féminine, tout en mélangeant subtilement, toutefois, le masculin et le féminin.

\section{La Common Law, union du féminin et du masculin?}

\section{La Common Law comparée à des figures masculines}

s'accorderait avec lesprt de conquête et de politique aviée qui caracté (1532), ce qui s'accorderait avec l'esprit de conquête et de politique avisée qui caractérise la Common Law. Dans l'œuvre de Machiavel, le Prince se doit d'« entretenir l'amitié des rois et des princes de sorte qu'ils aient ou plaisir à vous être utiles ou inquiétude à vous nuire " $(1992,97)$, et il est précisé que : «C'est par un combat sans fin que se conquiert et se maintient la liberté » $(1992,20)$. Cela peut faire écho au dernier Titre des conférences de Pollock intitulé : "The Perpetual Quest ", qui fait référence, précisément, à la quête incessante de la liberté.

Enfin, troisième et dernier exemple dans lequel féminin et masculin se mêlent lorsqu'il est question de la Common Law : c'est justement dans ce Titre qu'est citée l'Hydre de Lerne, qui symbolise l'excès de formalisme contre lequel la Common Law se bat. Or, dans la mythologie, c'est Hercule qui en est venu à bout, c'est-à-dire un homme, de surcroît doué de pouvoirs extraordinaires.

Les genres masculin et féminin semblent bel et bien mélangés. Pour autant, la Common Law serait-elle hermaphrodite?

Miranda, 12 | 2016 


\section{La Common Law, hermaphrodite?}

L'hypothèse ne semble pas hasardeuse si l'on prend en compte la distinction entre, d'une part, l'hermaphrodite en tant que concept, qui peut symboliquement être représenté comme un être divin et, d'autre part, l'hermaphrodite en tant que personne, c'est-à-dire comme un être incarné. La réponse à la question posée en sous-titre dépend de cette distinction, car la perception de l'un (le concept) et de l'autre (l'être incarné) est radicalement différente, et même totalement opposée.

Je vais m'appuyer ici sur trois ouvrages de référence : celui de Delcourt, Hermaphrodite (1958) ; celui de Eliade, Méphistophélès et l'androgyne (1962); et, enfin, celui de Libis, Le mythe de l'androgyne (1980).

Delcourt énonce très clairement l'ambivalence concept/personne dans le monde grec antique, lorsqu'elle oppose l'hermaphrodite en tant que concept, qui est « chargée de hautes valeurs » (68), alors que l'hermaphrodite incarné en un être humain est « une monstruosité » (même page). Ce point de vue est repris par Libis lorsqu'il indique que l'hermaphrodite est alors « objet de scandale, voire d'opprobre » (169). L'être de chair et de sang est une aberration de la nature et subit un rejet total: on brûle le monstre, ou bien on l'abandonne sur une île déserte, et l'on expose les nouveau-nés (aucun contact avec le corps ne doit avoir lieu); « [...] l'enfant qui naît avec les signes des deux sexes », explique Delcourt, « est maléfique et condamné à mort » (77).

Sur le plan anatomique, la question d'une similitude entre la Common Law et l'hermaphrodisme appelle une réponse clairement négative, étayée par trois éléments.

Le premier est qu'aucune caractéristique physique qui pourrait faire penser à l'hermaphrodisme n'est donnée par Pollock, qui ne s'étend d'ailleurs pas outre mesure sur l'apparence physique de la Common Law. Or, un hermaphrodite est justement défini par son apparence physique : il possède, selon le Dictionnaire alphabétique et analogique de la langue française, "quelques uns des caractères apparents des deux sexes ». Cela ne correspond donc en rien à l'image de la Common Law que l'on peut se faire en lisant Pollock.

Le deuxième est que l'hermaphrodite est stérile (en tous les cas, chez les espèces chez lesquelles il s'agit d'une erreur du programme génétique). Or, la Common Law est très féconde. Cette stérilité se retrouve également sur le plan symbolique : chez les Latins, mais aussi les Grecs, les Indiens d'Inde et les Égyptiens de l'Égypte ancienne, "l'indécision sexuelle des dieux est chose fréquente », explique Libis à la page 37 de son ouvrage, et ces dieux hommes/femmes sont toujours stériles, poursuit-il.

44 Enfin, Hermaphrodite était un jeune homme, or la Common Law est une femme d'un âge respectable, bien qu'elle ait conservé toute la vigueur de la jeunesse.

La fusion, voire la confusion, des apparences masculine et féminine, ne peut pas exister dans la Common Law telle que la décrit Pollock, sinon tout l'édifice sur lequel il a bâti son image s'écroule. La Common Law, sous sa plume, n'est certes pas sexualisée, mais elle est sexuée, puisque c'est clairement une femme. Aucun doute n'est possible sur ce point. Comme elle ne doit pas être objet de désir, mais objet de respect, Pollock en fait une femme sacralisée dont il gomme les caractéristiques physiques afin qu'elle ait l'air asexuée. 

l'unique élément susceptible d'établir un lien entre l'hermaphrodisme et la Common Law. C'est ce que nous apprend la légende d'Hermaphrodite et de la nymphe du lac, qui après s'être étroitement enroulée autour de lui a supplié les dieux de ne plus les séparer. Voici ce que Delcourt conclut : «Sous une double forme, ils ne sont ni homme ni femme; ils semblent n'avoir aucun sexe et les avoir tous les deux» (80). Or, symboliquement, la Common Law semble n'avoir aucun sexe, puisque Pollock la compare sans cesse à des figures sacrées désexualisées ; mais elle semble aussi les avoir tous les deux lorsqu'elle est comparée à des figures masculines, d'une part, et lorsqu'elle est associée à la guerre, à la violence et à la conquête qui procèdent, dans notre inconscient collectif, du principe masculin.

ce sens, elle est le lieu d'une réunion du féminin et du masculin, c'est-à-dire un être double qui semble bien posséder tous les attributs de cette bivalence. Premièrement, l'auto-procréation (selon Delcourt, l'hermaphrodite «se féconde et s'engendre luimême ", 110) ; or, la Common Law engendre ses propres enfants car le système est conçu ainsi, non sans le concours des juges (qui, durant plusieurs siècles, étaient exclusivement des hommes). Deuxièmement, la puissance, car la Common Law semble posséder les pouvoirs des deux sexes : sur le plan symbolique, la «femme virile » est, d'après Libis, "dotée de pouvoirs redoutables" (115); enfin, cet auteur indique également que l'hermaphrodite est associé au savoir (121), à la longévité, voire à l'immortalité, puisqu'elle/il est capable d'auto-procréation. Or, le système de Common Law est justement qualifié de " permanent » par Pollock (9). J'ai, à cet égard, proposé un lien avec la métaphore de l'arbre, également communément utilisée pour faire référence au système de Common Law, en montrant qu'elle peut symboliser une longévité éternelle (Richard 2014, 109).

\section{Conclusion}

Que dire, au final, puisque « elle » est aussi un « il »: «le» ou « la » Common Law?

Legrand démontre, dans son article, l'absence de rigueur scientifique qui accompagne le choix de l'article féminin devant le syntagme Common Law et pourfend, selon le même argument, la métaphore féminine de Pollock $(1992,945)$. Rigoureuse ou non, cette métaphore a néanmoins marqué son temps et les temps qui ont suivi : la Common Law semble bien avoir acquis un genre, pour ce qui est du référent symbolique auquel elle renvoie.

Pour autant, le genre grammatical n'étant pas nécessairement en accord avec l'objet qu'il désigne, cela signifie que rien ne pourrait empêcher d'utiliser l'article masculin devant un nom qui renvoie à un référent féminin. Ainsi, même si l'on remarque un lien entre genre grammatical et genre sexué, ce n'est pas un fait systématique. Par exemple, "une antilope » désigne aussi bien les individus femelles que mâles; en anglais, duck désignait les canes et drake les canards jusqu'à ce que le premier ait fini par englober le signifiant du second par extensivité (Tellier, 1962). De plus, le genre est fonction des langues : certaines en ont trois (masculin, féminin, neutre par exemple), d'autres en ont deux, et d'autres encore n'en ont pas du tout. De surcroît, ce genre peut varier d'une langue à l'autre pour un même référent (coche est masculin en espagnol alors que Kutsche est féminin en allemand; or, ces deux noms qui renvoient au même référent, ont la même étymologie ${ }^{11}$ ). 
Common Law pourrait faire partie de ces noms et syntagmes dits « hermaphrodites », ou encore "épicènes ». "Ce mot est un hermaphrodite : il a deux genres » indique, par exemple, le Dictionnaire cité plus haut. Ces mots, non marqués du point de vue du genre, peuvent de ce fait s'accommoder d'un article masculin comme d'un article féminin, en fonction de leur référent (comme "pédiatre ", " archéologue ", " élève ", « adulte »...). Cependant, Legrand s'oppose avec véhémence à cette flexibilité du genre, qu'il assimile à de l'anarchie ( Je n'accepte pas que l'absence de genre grammatical en langue anglaise, voire le manque de rigueur dont on a pu faire preuve en law French pendant des siècles, devraient impliquer l'anarchie en langue française ", déclare-t-il page 946). Cette remarque est tout à fait exacte: le choix de l'article ne devrait pas être anarchique. Pour autant, ce n'est pas une raison pour fermer la porte au féminin. Le choix en question devrait être éclairé, c'est-à-dire dicté par le référent de Common Law, qui peut être soit masculin, soit féminin. "Soit» dans le sens où ce peut être indécidable hors contexte, et parfois peut-être même en contexte.

51 Étant donné la force de la métaphore forgée par Pollock (bien que son propre fils l'ait qualifiée de "pleasant conceit », commente Cosgrove à la page 150 de son ouvrage), la prise en compte de la représentation féminine n'est pas moins légitime que celle de «droit jurisprudentiel ». Certains contextes vont même exiger le choix du féminin: comment traduire les conférences de Pollock autrement qu'en ayant recours à l'article féminin ? Il en va de même des nombreux ouvrages qui font référence, dans leur titre ou dans leur contenu, à « Our Lady the Common Law ». Contrairement à Legrand, je ne pense pas que l'on puisse trancher en la matière, car la matière est mouvante. J'en reviens à l'oxymore métaphorique du titre de cet article: «Le Common Law est une femme ». Une femme qui a absorbé et fait siens des principes dits masculins, érigeant un nouveau genre qui concentre en une seule entité les catégories « hommes »/ « femmes », tout en demeurant résolument «femme». Cette femme, qui est aussi un «il» par certains aspects, se trouve au centre de la rhétorique de la jurisprudence anglaise. Ainsi, l'article masculin que je propose n'est donc un paradoxe qu'en apparence: Le « Common Law » est une femme.

\section{BIBLIOGRAPHIE}

Baker, John Hamilton. Manual of Law French. Aldershot : Scolar Press, 1990.

Bullier, Antoine. La Common Law. 2ème éd. Paris : Dalloz, 2007.

Cosgrove, Richard A. Our Lady the Common Law - An Anglo-American Legal Community 1870-1930. New York \& Londres : New York University Press, 1987.

Delcourt, Marie. Hermaphrodite. Mythes et rites de la bisexualité dans l'Antiquité classique. 1958. Paris : Presses Universitaires de France, 1992.

Eliade, Mircea. Méphistophélès et l'androgyne. Paris : Gallimard, 1962.

Eschyle. Les Euménides. Paris : Belles Lettres, 1989. 
Galonnier, Bernard. « La métaphore dans les jugements anglais : nature et fonction » ASp 27-30 (2000) : 325-335. Avril 2013. <http://ASp.revues.org/2260>, 1-10.

Homère. L'Iliade. Paris : Garnier, 1977.

Kerby, Jean. « La traduction juridique, un cas d'espèce ». In Langage du droit et traduction, essais de jurilinguistique, Dir. Jean-Claude Gémar. Québec : Conseil de la langue française \& Linguatech, 1982. 3-10.

Laws, Lord Justice. « Our Lady of the Common Law ». Conférence donnée à la Law Society le 1 mars 2012. 5 juin 2013. <http://www.judiciary.gov.uk/media/speeches/2012/lj-laws-speech-iclrlecture-01032012>.

Legrand, Pierre. «Pour le common law », Revue internationale de droit comparé. Volume $44: 4$, (octobre-décembre 1992) : 941-947. 15 avril 2013. <http://www.persee.fr/web/revues/home/ prescript/article/ridc_0035-3337_1992_num_44_4_4577 >.

Libis, Jean. Le Mythe de l'androgyne. Paris : Berg International Éditeurs, 1980.

Machiavel. Le Prince. 1980. Paris : Flammarion, 1992.

Picotte, Jacques. Le Juridictionnaire. 1991. Bureau de la Traduction du Canada et Centre de Traduction et de Terminologie Juridiques (CTTJ). <http://www.cttj.ca/documents/ juridictionnaire.pdf> 2014 .

Pollock, Frederick. The Genius of the Common Law. New York : Columbia University Press, 1912.

Pollock Frederick \& Maitland, Frederic William. The History of English Law, vol.1. 1895淿

Cambridge : Cambridge University Press, 1968.

Richard, Isabelle. « Metaphors in English for Law : Let Us Keep Them! », Lexis 8 Metaphors Studies in the English Language (2014), 103-122. <http://lexis.univ-lyon3.fr/spip.php?article203>.

Robert, Paul. Dictionnaire alphabétique et analogique de la langue française. Paris : Le Robert, 1974.

Tellier, André. Les verbes perfecto-présents et les auxiliaires de mode en anglais ancien ( $8^{\text {ème }}-16^{\text {ème }}$ siècles), thèse pour le Doctorat ès Lettres. Paris : Klincksieck, 1962.

Watt, Gary. Equity Stirring - The Story of Justice Beyond Law. 2009. Oxford and Portland, Oregon : Hart Publishing, 2012.

\section{NOTES}

1. Pollock était un Barrister, auteur avec Maitland du célèbre ouvrage History of English Law Before the Time of Edward the First (1895).

2. Benjamin Cardozo a été nommé juge à la Cour suprême des États-Unis en 1932.

3. Les lois ont été rédigées en Law French du $12^{\text {ème }}$ au $15^{\text {ème }}$ siècles. Ce n'est qu'au $18^{\text {ème }}$ siècle, toutefois, que le Parlement a imposé l'emploi de l'anglais dans l'ensemble de la pratique juridique, de sorte que tous les documents juridiques devaient être rédigés dans cette langue, $\mathrm{y}$ compris les recueils de jurisprudence.

4. «By the end of the year-book period [c'est-à-dire vers 1536] the definite article was not even an indicator of sex [...]. [...] Plowden, in the mid-sixteenth century [c'est-à-dire en 1571], could write ma file and mon file, sa feme and le feme, on the same page » écrit Legrand (1992, 944), citant Baker (1990, 13 , note 17$)$.

5. J'ai utilisé, pour cet article, la version des conférences de Pollock téléchargeable depuis le site Online Library of Liberty. Les numéros de pages cités sont donc ceux de ce document. 
6. Dans le cadre d'une procédure civile, surrebutter fait référence à un moyen de défense qui consiste à contredire ou à annuler une preuve avancée par le demandeur ; celui-ci peut répondre par le biais de surrebutter qui est donc, en quelque sorte, une contre-attaque à une contreattaque.

7. http://dreamstime.com/phtographie-stock-libre-de-droits-ath\%C3\%A9na-d\%C3\%A9esse-dela-mythologie-grecque-image26085457.

http://www.nella-buscot.com/jardins_paris_7_palais_bourbon.php. (Bas de page) http://alyabbara.com/histoire/Mythologie/grecce/images/Athena_Damas_Syrie_detail.html.

8. http://remacle.org/bloodwolf/tragediens/eschyle/eumenides.htm.

9. Cette règle a été introduite pour la première fois en 1615 par James I, et confirmée par les Judicature Acts des années 1870.

10. On peut faire un rapprochement avec Domestic Law.

11. Ces deux substantifs viennent du hongrois kocsi signifiant "voiture de Kocs» (coach en est également dérivé) ; il s'agissait d'une carriole à quatre roues montée sur ressorts, tirée par des chevaux et originaire de Kocs, un village situé au nord-ouest de Budapest. Le hongrois ne connaissant pas le genre grammatical, cela pourrait expliquer que l'espagnol ait opté pour le masculin et l'allemand pour le féminin.

\section{RÉSUMÉS}

Sir Frederick Pollock (1845-1937), célèbre historien du droit anglais et spécialiste du Common Law, a donné en 1911, à l'université de Columbia (New York), une série de conférences intitulées The Genius of the Common Law, qui ont créé une personnification féminine de ce système de droit. Cette féminisation semble, depuis, s'être ancrée dans l'imaginaire des juristes des deux côtés de l'Atlantique, comme en attestent un certain nombre d'études et de discours parus depuis. Une autre source majeure du droit anglais, Equity, est également associée à des images féminines. Cette féminisation d'aspects fondamentaux du droit anglais construit une rhétorique au sein de laquelle ces représentations imposent une certaine image du féminin. Cet article est fondé sur l'analyse des conférences de Sir Pollock, que viendront compléter certains aspects de l'ouvrage de Gary Watt, Equity Stirring - The Story of Justice Beyond Law (2012). Il discutera, notamment, du lien grammatical qui peut, ou non, se faire en français avec ces représentations féminines lorsque nous utilisons le terme Common Law.

Sir Frederick Pollock (1845-1937), a leading English historian and a specialist of the Common Law, gave a series of lectures at the University of Columbia (New York) in 1911. Entitled The Genius of the Common Law, they initiated a female personification of this system of law which, to all appearances, has strongly influenced the way lawyers from both sides of the Atlantic think of the law, as is shown by a number of books, articles and speeches published since. Besides, the other main source of case law, Equity, is also referred to in terms of feminine metaphors. This feminisation of both Common Law and Equity has generated a legal rhetoric based on specific feminine figures. This article will analyse Sir Pollock's lectures as well as certain aspects of Gary Watt's Equity Stirring - The Story of Justice Beyond Law (2012) and will discuss the grammatical link that should or should not be made with these figures when we use the term "Common Law" in French. 
INDEX

Keywords : Common Law, Equity, gender, feminisation, translation

Mots-clés : Common Law, Equity, genre, féminisation, traduction

\section{AUTEURS}

\section{ISABELLE RICHARD}

Maître de conférences HDR

Aix-Marseille Université

isabelle.richard@univ-amu.fr 